\title{
Chiral Lead Halide Perovskite Nanowires for Second-Order Nonlinear Optics
}

\author{
Chunqing Yuan, ${ }^{\dagger}$ Xinyue Li, ${ }^{\dagger, \ddagger}$ Sergey Semin, ${ }^{\ddagger}$ Yaqing Feng, ${ }^{\dagger}$ Theo Rasing, ${ }^{\ddagger}$ and Jialiang Xu ${ }^{*},, \S_{0}$ \\ ${ }^{\dagger}$ School of Chemical Engineering and Technology, Tianjin University, Yaguan Road 135, Tianjin 300350, P.R. China \\ ${ }^{*}$ Institute for Molecules and Materials (IMM), Radboud University, Heyendaalseweg 135, 6525AJ Nijmegen, The Netherlands \\ ${ }^{\S}$ School of Materials Science and Engineering, Nankai University, Tongyan Road 38, Tianjin 300350, P.R. China
}

\section{Supporting Information}

ABSTRACT: Hybrid organic/inorganic lead halide perovskites (LHPs) have recently emerged as extremely promising photonic materials. However, the exploration of their optical nonlinearities has been mainly focused on the third- and higher-order nonlinear optical (NLO) effects. Strong secondorder NLO responses are hardly expected from ordinary LHPs due to their intrinsic centrosymmetric structures, but are highly desirable for advancing their applications in the next generation integrated photonic circuits. Here we demonstrate the fabrication of a novel noncentrosymmetric LHP material by introducing chiral amines as the organic component. The nanowires grown from this new LHP

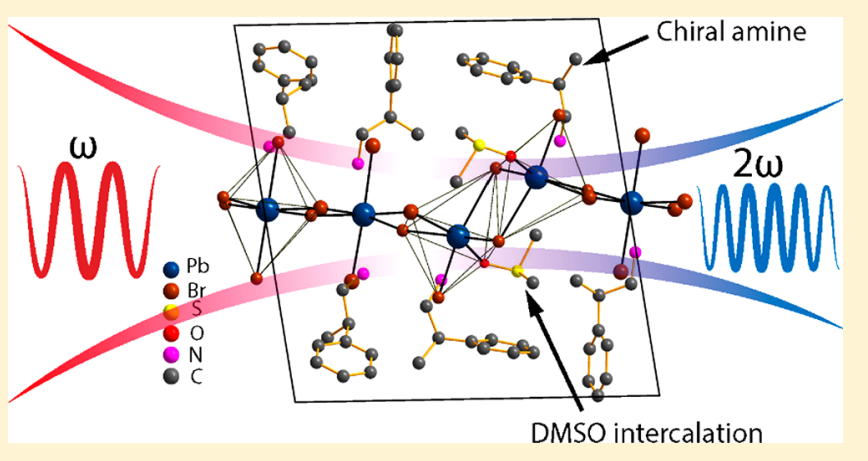
material crystallize in a noncentrosymmetric $P 1$ space group and demonstrate highly efficient second harmonic generation (SHG) with high polarization ratios and chiroptical NLO effects. Such a chiral perovskite skeleton could provide a new platform for future engineering of optoelectronic functionalities of hybrid perovskite materials.

KEYWORDS: Chiral perovskite, nanowire, nonlinear optics, second harmonic generation

$\mathrm{H}$ ybrid organic/inorganic lead halide perovskites (LHPs), featuring intrinsic chemical variability and structural diversity, ${ }^{1}$ have recently emerged as extremely promising photonic materials $^{2-4}$ for diverse optoelectronic device applications in light emitting diodes, ${ }^{5,6}$ solar cells, ${ }^{7,8}$ photoelectrolysis, ${ }^{9}$ photodetectors, ${ }^{10,11}$ and optically pumped lasers. ${ }^{12}$ The nonlinear optical (NLO) effects are fundamentals for realizing some very important optical devices such as optical parametric generators and oscillators (OPGs and OPOs respectively), all optical switches, optical limiters, and so on. ${ }^{13-15}$ Meanwhile, the NLO materials are also widely applied in advanced techniques such as high resolution optical lithography ${ }^{16}$ and microscopy. ${ }^{17}$ Perovskite-type materials such as niobates, ${ }^{18,19}$ borates, $^{20}$ titanates, ${ }^{21,22}$ germanium iodides, ${ }^{23}$ etc. have been widely explored as NLO materials for their versatile chemical structures and large NLO coefficients. In particular, the hybrid organic/inorganic LHPs allow for enormous opportunities to fine-tune the structural scaffolds and electronic bandgaps of perovskite materials by varying the organic and inorganic components. Along with the merits of low-cost and processability, they are therefore very promising for applications in NLO materials. The third- and higher-order NLO properties of LHP materials have been extensively investigated and widely applied in optical devices such as upconversion lasers. ${ }^{24-27}$ However, for electro-optical and frequency doubling/mixing applications, second-order NLO effects are required. ${ }^{28}$ For second-order NLO responses such as second harmonic generation (SHG), a strict structural noncentrosymmetry is required. ${ }^{29}$ Although weak, presumably surface related SHG signals have been detected from microdomains of tetragonal $\mathrm{CH}_{3} \mathrm{NH}_{3} \mathrm{PbI}_{3}$ based LHP films, ${ }^{30}$ strong second-order NLO responses have been hardly observed from the bulk of the ordinary LHP materials, consistent with their centrosymmetric crystal space groups. ${ }^{25,31}$ In addition, second-order optical nonlinearities are often accompanied by some other technologically important effects such as ferroelectricity, pyroelectricity and piezoelectricity, because these effects also require structural noncentrosymmetry. A few hybrid LHP materials have indeed been demonstrated to be superior ferroelectric semiconductors. ${ }^{32,33}$ In this context, a rational design and construction of LHPs with intrinsic noncentrosymmetric geometries and bulk second-order optical nonlinearities is of particular importance, not only for advancing their applications in the next generation integrated photonic circuits, but also for providing a prototype for developing new LHP functional materials and devices. In particular, the elongated one-dimensional nanostructure of these LHP materials makes them potential building blocks for nanowire photonics. ${ }^{34,35}$

Received: April 21, 2018

Revised: July 30, 2018

Published: August 13, 2018 
Scheme 1. Crystallographic Structure of the Chiral Perovskites As Obtained from Single Crystal XRD Measurements, Showing the Features of Chirality, DMSO Intercalation, and Partial Edge Sharing ${ }^{a}$

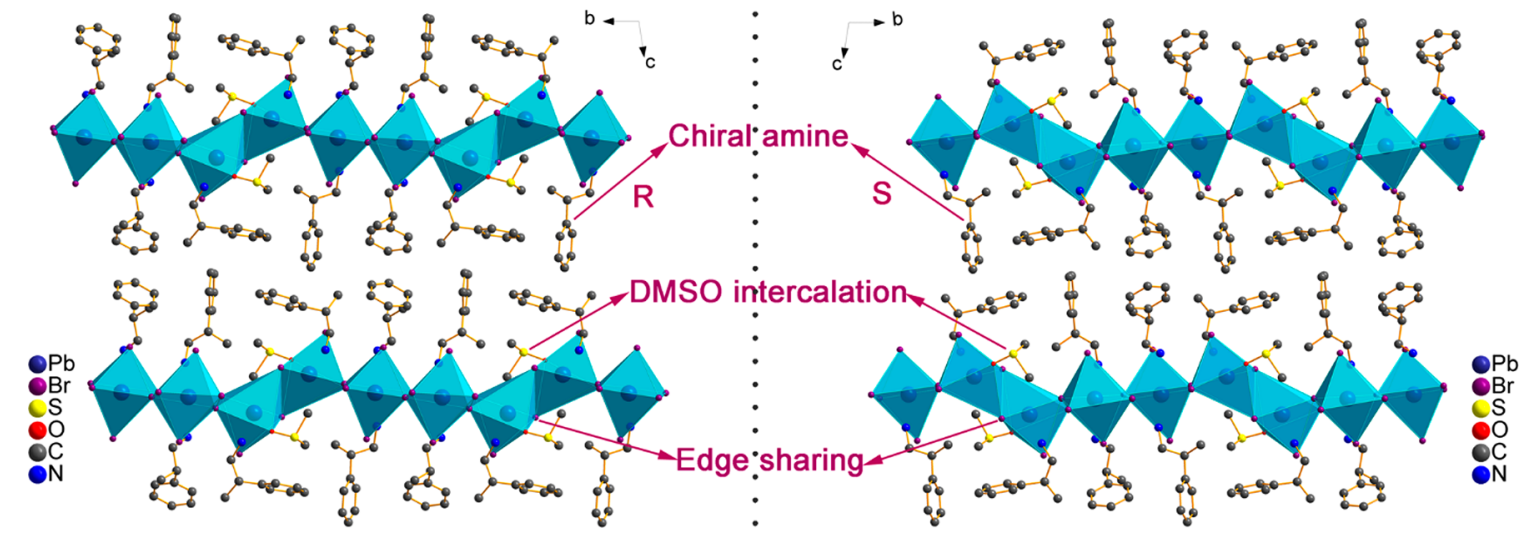

${ }^{a_{T}}$ The chemical formulas were determined to be (R-MPEA $)_{1.5} \mathrm{PbBr}_{3.5}(\mathrm{DMSO})_{0.5}$ and $(S-\mathrm{MPEA})_{1.5} \mathrm{PbBr}_{3.5}(\mathrm{DMSO})_{0.5}$, respectively. $\mathrm{H}$ atoms are omitted for clarity.

Recently, two-dimensional (2D) hybrid LHP materials with relatively bulky organic motifs have attracted broad interests for their improved stability under ambient conditions. The bulky organic site has provided a versatile platform for the functionalization of these $2 \mathrm{D}$ perovskite materials by introducing diverse ammonium groups for superior optoelectronic properties. ${ }^{36-39}$ Billing and Lemmerer, ${ }^{40}$ and Moon et al. $^{41}$ have also demonstrated the feasibility of introducing chirality into LHP materials by employing chiral amine as the organic component. Inspired by these pioneering works, we report here the construction of a new chiral LHP material and the observation of strong bulk second-order NLO responses from nanowires of this chiral perovskite material. Chiral $\beta$ methylphenethylamine (MPEA) was used as the organic component to direct the noncentrosymmetric assembly of the $2 \mathrm{D}$ inorganic layers and perovskite crystals into a chiral $P 1$ space group, by antisolvent vapor-assisted crystallization $(\mathrm{AVC})^{42}$ using a ternary solvent system. The structure of the dimethyl sulfoxide (DMSO) solvent engineered 2D perovskite intermediate phase was determined to be a new type of perovskite material in which DMSO molecules axially coordinated with $\mathrm{Pb}^{2+}$ in the partially edge-shared octahedrons with a formula of (MPEA $)_{1.5} \mathrm{PbBr}_{3.5}(\mathrm{DMSO})_{0.5}$. Nanowires from this chiral perovskite material were fabricated and strong SHG signal was observed from the nanowires with high polarization ratios and NLO circular dichroism. This chiral perovskite skeleton offers a new platform for future engineering of the optoelectronic and other functionalities of hybrid perovskite materials.

Results and Discussion. The chiral perovskite materials were obtained by incorporating the bulky $(R)-(+)$ - or $(S)$ $(-)-\beta$-methylphenethylammonium cation $(R-$ or $S$-MPEA) into the $2 \mathrm{D}$ perovskite scaffolds. We followed the AVC technique for the growth of the chiral perovskite single crystals with chloroform $\left(\mathrm{CHCl}_{3}\right)$ as the antisolvent and $\mathrm{N}, \mathrm{N}$ dimethylformamide (DMF) as the good solvent ${ }^{42}$ (see details in the Supporting Information). The slow diffusion of $\mathrm{CHCl}_{3}$ vapor into the mixed solution of $\mathrm{PbBr}_{2}$ and $\mathrm{MPEA}^{+} \mathrm{Br}^{-}$ precursors in DMF results in the growth of white needlelike single crystals (Supporting Information Figure S1). However, these needle crystals are small and of poor quality, which makes them unsuitable for single crystal X-ray diffraction (SCXRD) analysis. We therefore sought a ternary solvent system in which a mixture of DMF and DMSO was utilized as the good solvent instead of pure DMF. ${ }^{43}$ The employment of DMSO indeed improved the crystal size and the optical quality of the as-formed single crystals (Figure S1), which made SCXRD measurements possible. Scheme 1 shows the crystallographic structures of the perovskite single crystals grown from the $R$ - and $S$-MPEA ${ }^{+} \mathrm{Br}^{-}$with $\mathrm{PbBr}_{2}$ precursors in this ternary solvent system (referred to as the $\alpha$-phase), respectively. We identified that the structure of as-formed perovskite materials grown from the ternary solvent system is very different from that of the ordinary $2 \mathrm{D}$ perovskites ${ }^{36,39}$ or the chiral perovskites, ${ }^{40,41}$ both of which feature a chemical formula of $\mathrm{A}_{2} \mathrm{PbX}_{4}(\mathrm{~A}=$ ammonium cation, $\mathrm{X}=\mathrm{Cl}, \mathrm{Br}$, or $\mathrm{I})$. In our case, the solvent DMSO is directly involved in the structure of the $\alpha$-phase perovskite crystal, resulting in layered 2D perovskite materials with chemical formulas of $(R$ M P E A ) ${ }_{1.5} \mathrm{P} \mathrm{b} \mathrm{B} \mathrm{r}_{3.5}(\mathrm{D} \mathrm{M} \mathrm{S} \mathrm{O})_{0.5}$ a n d $\quad(S-$ MPEA $)_{1.5} \mathrm{PbBr}_{3.5}(\mathrm{DMSO})_{0.5}$, respectively (Table S1). Seok et al. reported the feasibility of the inclusion of DMSO to improve the quality of spin-coated perovskite films for enhanced photovoltaic performances, by forming an intermediate phase with DMSO molecules intercalated between the inorganic layers. ${ }^{43}$ However, the crystal structure of this intermediate phase has not been solved yet and the positions of the DMSO guest molecules are not clearly defined. In the case here, we identify that the molar ratio of the precursors in the "MPEA ${ }^{+} \mathrm{Br}^{-}-\mathrm{PbBr}_{2}$-DMSO" complex is $3: 2: 1$, and the DMSO molecules are involved in the crystal structure by axial coordination with the $\mathrm{Pb}^{2+}$ centers in the octahedron (Scheme 1 ). Half of the octahedrons in the crystal indeed share one of their edges with the neighboring octahedrons, to some extent similar to the proposed structures of the intermediate phase formed in the spin-coated films. ${ }^{43}$ However, we find that these DMSO complexed phases of our chiral perovskite crystals are actually very stable in ambient conditions. The thermogravimetric analysis (TGA) suggests that they do not undergo any clear weight loss until $141{ }^{\circ} \mathrm{C}$ (Figure S2), comparable to that of the perovskite crystals grown from pure DMF (referred to as the $\beta$-phase) and other ordinary 2D hybrid LHP materials. ${ }^{44,45}$ Nevertheless, the introduction of the chiral ligand R-MPEA induces these perovskite materials to crystallize in an intrinsically chiral and noncentrosymmetric triclinic $P 1$ space group (point group: 1), which is of crucial importance for the 
a

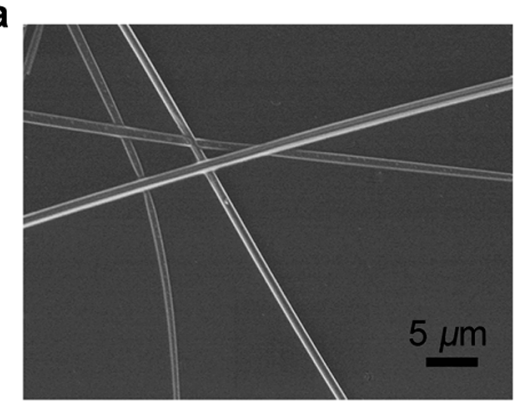

C

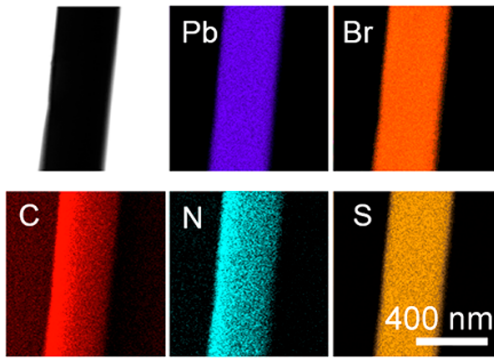

b

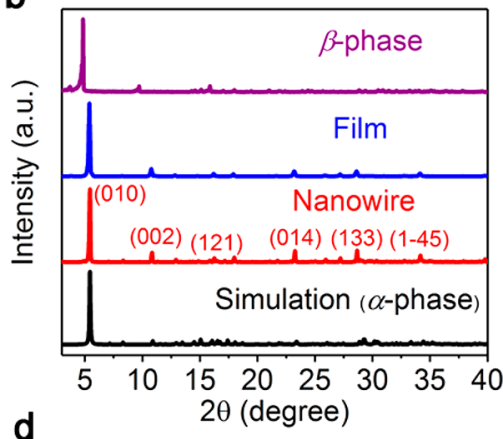

d

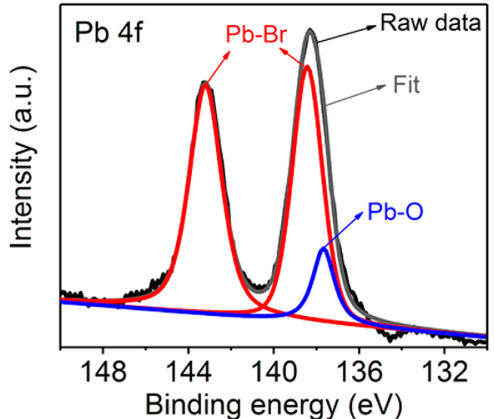

Figure 1. Characterizations of the chiral perovskite nanowires. (a) SEM image of the $(R-M P E A)_{1.5} \mathrm{PbBr}_{3.5}(\mathrm{DMSO})_{0.5}$ nanowires. (b) PXRD patterns of the as-grown (R-MPEA $)_{1.5} \mathrm{PbBr}_{3.5}(\mathrm{DMSO})_{0.5}$ nanowires, in comparison with the simulated XRD pattern from the single crystal structures, and those measured from the spin-coated films and the $\beta$-phase crystals. The PXRD patterns of the nanowires resemble the simulated patterns from the $\alpha$-phase chiral perovskite single crystals well, revealing that they belong to the same crystal phase and have the same crystallographic structures as shown in Scheme 1. (c) Typical TEM image of an individual $\left(R-\mathrm{MPEA}_{1.5} \mathrm{PbBr}{ }_{3.5}(\mathrm{DMSO})_{0.5}\right.$ nanowire, and the corresponding EDX mappings. (d) Decomposed high-resolution XPS spectrum of $\mathrm{Pb} 4 \mathrm{f}$ for the $(\mathrm{R}-\mathrm{MPEA})_{1.5} \mathrm{PbBr}{ }_{3.5}(\mathrm{DMSO})_{0.5}$ nanowires.

observation of a bulk second-order nonlinear optical response. The single crystal structures of both ( $R$ M P E A ) ${ }_{1.5} \mathrm{P} \mathrm{b} \mathrm{B} \mathrm{r}_{3.5}(\text { D M S O })_{0.5}$ a n d ( $S$ MPEA $)_{1.5} \mathrm{PbBr}_{3.5}$ (DMSO) $)_{0.5}$ belong to the same space group with almost identical unit cell parameters (Table S1) and very similar bond lengths and angles (Table S2). However, they have mirror symmetry geometries (Scheme 1), as expected from the enantiomers.

We fabricated high quality nanowires of the chiral perovskites by liquid diffusion instead of the AVC vapor diffusion method, with the same ternary solvent system (see the Supporting Information). The liquid diffusion of the antisolvent $\mathrm{CHCl}_{3}$ into the solution of the mixed precursors $\left(R-\mathrm{MPEA}^{+} \mathrm{Br}^{-}\right.$and $\left.\mathrm{PbBr}_{2}\right)$ in the combined solvent of DMF and DMSO results in nanowires of the chiral perovskites suspended in the final ternary solvent mixture. Scanning electron microscopy (SEM) images of the as-prepared samples show that the nanowires typically have diameters of hundreds of nanometers and lengths of up to hundreds of micrometers (Figures 1a and S3). The powder X-ray diffraction (PXRD, Figure $1 \mathrm{~b}$ ) patterns of the as-prepared nanowires resemble the simulated patterns from the $\alpha$-phase chiral perovskite single crystals, revealing that these nanowires grown with liquid diffusion belong to the same crystal phase as that of the bulk chiral perovskite crystals grown by the AVC technique. That is, the nanowires also belong to the noncentrosymmetric triclinic $P 1$ space group, and feature structural chirality, DMSO intercalation, and partial edge sharing as shown in Scheme 1. The comparison of the PXRD patterns also shows that this $\alpha$ phase chiral perovskite grown in the ternary solvent system is clearly different from that of the $\beta$-phase perovskite crystals precipitated from DMF.
The energy dispersive X-ray spectroscopy (EDX) mapping of a typical individual nanowire under the transmission electron microscope (TEM) reveals the coexistence of $\mathrm{C}, \mathrm{N}$, $\mathrm{Pb}$, and $\mathrm{Br}$ elements (Figure 1c), as expected from the corresponding organic and inorganic precursors. Meanwhile, the uniform elemental distribution of $S$ confirms the involvement of the solvent DMSO in the perovskite nanowire crystals. We further studied the nature of this involvement with X-ray photoelectron spectroscopy (XPS). The XPS spectrum of the as-prepared nanowires depicts the expected characteristic peaks of the hybrid organic/inorganic LHPs such as the bands of $\mathrm{C} 1 \mathrm{~s}$ (from both the $\mathrm{sp}^{2}$ and $\mathrm{sp}^{3} \mathrm{C}-\mathrm{C}$ bonds as well as the $\mathrm{C}-\mathrm{N}$ bond; see Figure $\mathrm{S} 4$ ), N 1s, $\mathrm{Br} 3 \mathrm{~d}, \mathrm{~Pb} 4 \mathrm{f}$, and so on. The decomposed high resolution XPS spectrum of the $\mathrm{Pb}$ $4 \mathrm{f}$ band demonstrates the presence of not only $\mathrm{Pb}-\mathrm{Br}$, but also the $\mathrm{Pb}-\mathrm{O}$ bond (Figure $1 \mathrm{~d}$ ). The presence of the $\mathrm{Pb}-\mathrm{O}$ bond is in accordance with the axial coordination of the incorporated guest $\mathrm{DMSO}$ molecules with the $\mathrm{Pb}^{2+}$ centers in the octahedron, as demonstrated by the single crystal structure of the bulk crystals.

We first studied the linear optical properties of these chiral perovskite materials using circularly polarized light to obtain circular dichroism (CD) spectra. We prepared high quality films of these perovskite materials for the $C D$ measurements by spin-coating the DMF solutions of the as-prepared $R$ - and $S$-(MPEA $)_{1.5} \mathrm{PbBr}_{3.5}(\mathrm{DMSO})_{0.5}$ crystals, respectively (see the Supporting Information for the preparation of perovskite films). We first checked the PXRD patterns of these films and found they retained the structure of the AVC grown $\alpha$-phase single crystal (Figure 1b). The thickness of the films was $~ 350$ $\mathrm{nm}$ as determined by the $3 \mathrm{D}$ graphic profile from the surface topography measurements (Figure S5), and the CD spectra of 

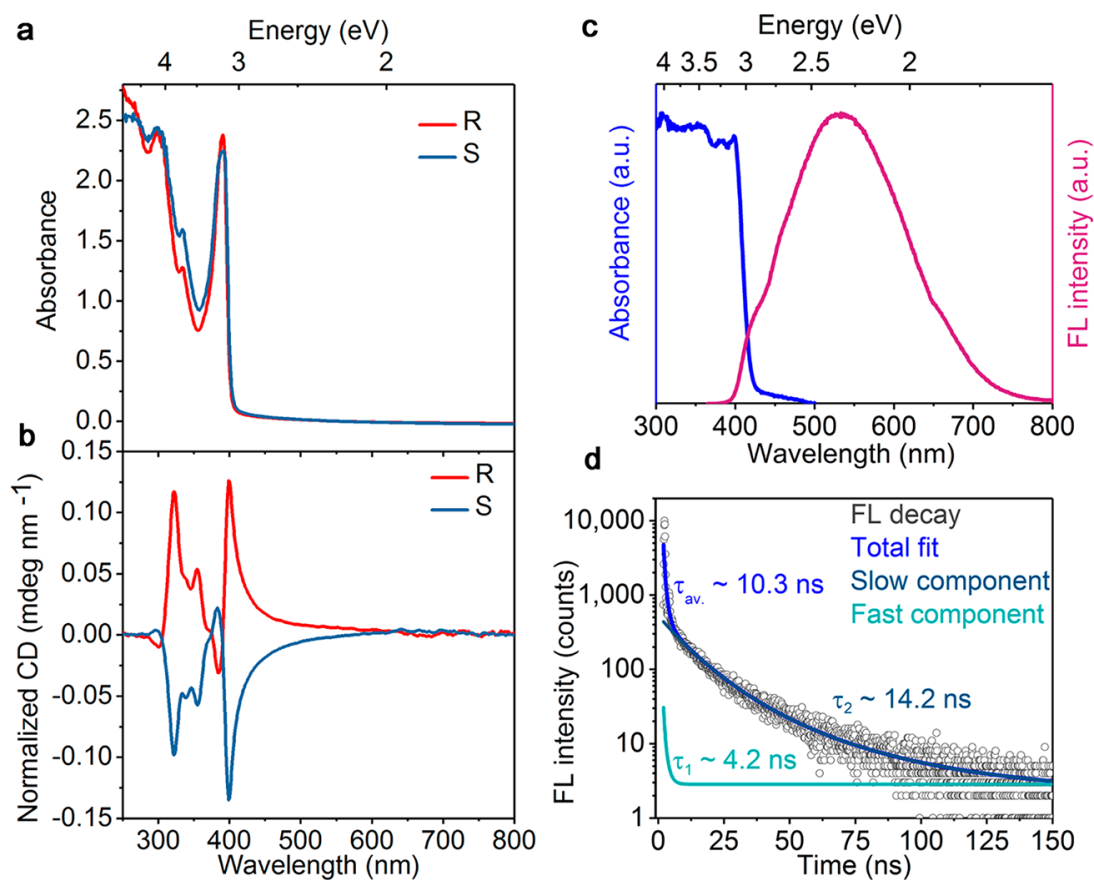

Figure 2. Linear optical properties of the chiral perovskite materials. UV-vis absorbance (a) and normalized CD (b) spectra of the films spincoated from DMF solutions of the $R$ - and $S$-(MPEA $)_{1.5} \mathrm{PbBr}_{3.5}(\mathrm{DMSO})_{0.5}$ perovskite crystals. Average film thickness for both $R$ - and $S$ $(\mathrm{MPEA})_{1.5} \mathrm{PbBr}_{3.5}(\mathrm{DMSO})_{0.5}$ is $\sim 350 \mathrm{~nm}$. (c) Normalized DRS and fluorescence spectra of the as-prepared $\left(R-\mathrm{MPEA}_{1.5} \mathrm{PbBr}{ }_{3.5}(\mathrm{DMSO})_{0.5}\right.$ nanowires. Excitation wavelength: $400 \mathrm{~nm}$. (d) Fluorescence decay of the $(R-M P E A)_{1.5} \mathrm{PbBr}_{3.5}(\mathrm{DMSO})_{0.5}$ nanowires. Measured at $\lambda=530 \mathrm{~nm}$ with the excitation wavelength of $400 \mathrm{~nm}$ at room temperature.

these films were measured in the transmission geometry. To elucidate and exclude the orientation effects on the UV-vis absorbance and CD measurements, the spectra are taken by rotating the film sample at every $60^{\circ}$. The as-measured results as shown in Figure $\mathrm{S} 6$ show that the spectra taken at different rotation angles have similar spectral features, and the intensity variations between measurements are small $(<10 \%)$. Figure $2 \mathrm{a}, \mathrm{b}$ shows the average UV-vis absorbance and the corresponding CD spectra of these spin-coated films, respectively. Both the $R$ - and $S$-(MPEA $)_{1.5} \mathrm{PbBr}_{3.5}(\mathrm{DMSO})_{0.5}$ perovskite films exhibit strong $\mathrm{CD}$ signals peaked at 325 and $405 \mathrm{~nm}\left(0.12\right.$ and $0.14 \mathrm{mdeg} \mathrm{nm}^{-1}$, respectively, in the normalized CD spectra ${ }^{46}$ ), but with opposite signs. In contrast, the CD signals of the chiral $R$ - and $S$-MPEA amine precursors appear at 226 and $257 \mathrm{~nm}$, respectively (Figure S7). Apparently, the $\mathrm{CD}$ responses of the chiral perovskites result from the Cotton effects of the intrinsic excitonic absorbance bands of both the $R$ - and $S$-(MPEA) $)_{1.5} \mathrm{PbBr}_{3.5}(\mathrm{DMSO})_{0.5}$ perovskite materials, which have been measured to be at 390 $\mathrm{nm}(3.18 \mathrm{eV})$ from the absorption spectra of the films (Figure 2a) (band edge: $3.07 \mathrm{eV}$, Figure S8). It is also notable that the $\mathrm{CD}$ signals proceed at longer wavelengths $(>420 \mathrm{~nm})$, which are beyond the excitonic absorbance bands of the perovskite materials. These signals might be attributed to circular differential scattering (CDS), ${ }^{41}$ owing to the different degrees of scattering of these chiral perovskite films for left- and rightcircularly polarized light. ${ }^{47}$

We measured the UV-vis absorbance of the as-prepared perovskite nanowires with a diffusive reflectance spectrum (DRS, Figure 2c), from which we determined its optical band gap to be $\sim 3.07 \mathrm{eV}$ (Figure S8). Upon excitation at $400 \mathrm{~nm}$, the nanowire crystals exhibited a broad emission, spanning the entire visible range with a maximum at $528 \mathrm{~nm}$ and a large fullwidth at half-maximum (fwhm) of $177 \mathrm{~nm}(780 \mathrm{meV})$, similar to the optical characteristics of the corrugated $2 \mathrm{D}$ (EDBE)$\left[\mathrm{PbBr}_{4}\right]^{44}$ and the elongated 1D $\left(\mathrm{C}_{4} \mathrm{~N}_{2} \mathrm{H}_{14}\right)\left[\mathrm{PbBr}_{4}\right]^{45}$ lead bromide perovskites. Although not well-defined, a higherenergy shoulder peak is evident in the photoluminescence spectrum at $\sim 410 \mathrm{~nm}$. When this shoulder emission originates from the free excitons, the low energy broad main emission should be attributed to the self-trapped excitons. ${ }^{48-50}$ The real color fluorescence microscope image shows a yellowish white light emission from these nanowires (Figure S9). The Commission Internationale de l'Eclairage (CIE) chromaticity coordinate of the overall emission from the perovskite nanowires was determined to be $(0.40,0.42)$ with the correlated color temperature (CCT) of $\sim 3800 \mathrm{~K}$, corresponding to the "warm" yellowish white light emission (Figure S9). ${ }^{51}$

We investigated the dynamics of this broad band emission with time-resolved fluorescence spectra. The decay profile of the emission from the (R-MPEA $)_{1.5} \mathrm{PbBr}_{3.5}(\mathrm{DMSO})_{0.5}$ nanowires shows a biexponential fit with a fast $\left(\tau_{1} \sim 4.2 \mathrm{~ns}\right)$ and a slow $\left(\tau_{2} \sim 14.2 \mathrm{~ns}\right)$ transient and an average lifetime of $10.3 \mathrm{~ns}$ (Figure $2 \mathrm{~d}$ ). It is notable that the $\beta$-phase perovskite crystals grown from pure DMF also demonstrate a broadband emission peaked at $439 \mathrm{~nm}$, with a fwhm of $106 \mathrm{~nm}(605 \mathrm{meV})$ (Figure S10) and CIE chromaticity coordinate of $(0.18,0.21)$ (Figure S9). The average lifetime of this emission is $2.2 \mathrm{~ns}$ (Figure S11), which is significantly shorter than that of the $\alpha$-phase perovskite nanowires grown from the mixture of DMF and DMSO. Clearly, the incorporation of DMSO improves the properties of these chiral perovskite materials as a potential white light emitter. Future engineering of the perovskite structures, by tuning the composition of the halides for example, might ensure a better color purity and quantum efficiency of the broadband white light emission of these chiral perovskite materials for practical indoor illumination applications. $^{44}$ 
a

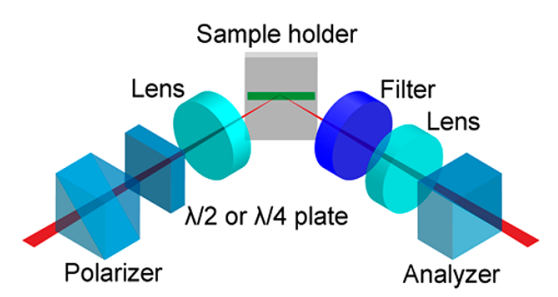

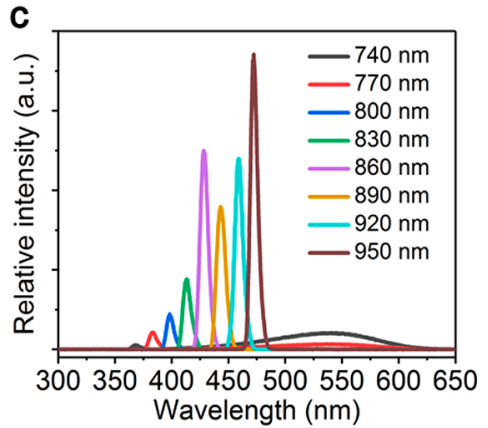

b

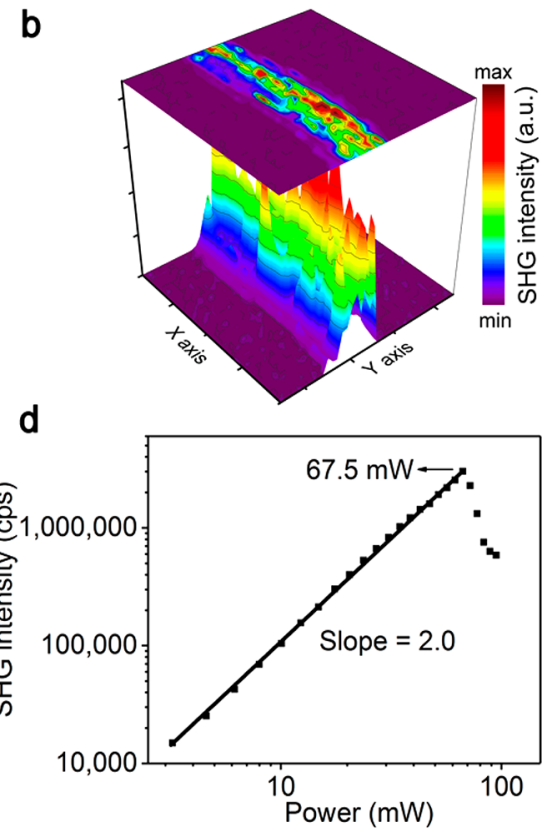

d

Figure 3. Nonlinear optical responses of the chiral perovskite nanowires. (a) Schematics of the NLO measurements. $\lambda / 2$ and $\lambda / 4$ plates were used for linearly and circularly polarized experiments, respectively. (b) Scanned image of a typical (R-MPEA $)_{1.5} \operatorname{PbBr}_{3.5}(\mathrm{DMSO})_{0.5}$ nanowire by detecting the SHG signal. Scanned area: $20 \times 20 \mu \mathrm{m}^{2}$. (c) NLO spectra of a $(R-M P E A)_{1.5} \mathrm{PbBr}_{3.5}(\mathrm{DMSO})_{0.5}$ nanowire pumped at various wavelengths, normalized by the incident power. (d) Logarithmic plot of SHG intensity as function of the incident power. The solid line is a linear fit with a slope of 2.0. The SHG intensity drops when the incidence power is higher than $67.5 \mathrm{~mW}$, showing the laser damage threshold. Excitation and detection wavelengths are 850 and $425 \mathrm{~nm}$, respectively, for panels (b) and (d).

We choose the (R-MPEA $)_{1.5} \mathrm{PbBr}_{3.5}(\mathrm{DMSO})_{0.5}$ nanowires as the model system for the study of the NLO properties of these new chiral perovskite materials. We utilized a home-built microscope equipped with a femtosecond pulsed laser as the pump, and drop-casted the as-grown nanowires into the suspension on the substrate for the NLO examinations (see details in the Supporting Information). We rotated the sample holder with the substrate containing the perovskite nanowires to ensure the selected single nanowire was parallel to the plane of incidence (Figure 3a). Figure $3 \mathrm{~b}$ shows the SHG image of a typical horizontally oriented nanowire, obtained by detecting the SHG signal (at $425 \mathrm{~nm}$ when pumped at $850 \mathrm{~nm}$ ) while scanning the sample. The image clearly shows the outline of the nanowire and demonstrates the second-order NLO activity of the chiral perovskite materials. The NLO spectra taken from the nanowire, shown in Figure $3 c$, demonstrate that the nanowire exhibits strong SHG signals when pumped at various wavelengths over the near-infrared (NIR) region. One can also clearly notice a broad FL peak when the excitation wavelength is below $800 \mathrm{~nm}$, which should be attributed to two-photon excited fluorescence (TPF), considering its energy upconversion characteristic. ${ }^{52}$ We evaluated the effective second-order NLO coefficient $\left(d_{e f f}\right)$ of the $\alpha$-phase $(R$ MPEA $)_{1.5} \mathrm{PbBr}_{3.5}(\mathrm{DMSO})_{0.5}$ perovskite material to be $\sim 0.68$ $\mathrm{pm} \mathrm{V}^{-1}$ with the optimized linearly polarized pump at $850 \mathrm{~nm}$ by using a Y-cut quartz as the reference (see details in the Supporting Information). This value compares favorably with that of potassium dihydrogen phosphate (KDP). ${ }^{53}$ Figure $3 \mathrm{~d}$ demonstrates a quadratic dependence of the SHG intensity on the incident laser power and confirms the two-photon nature of this second-order NLO response. However, the SHG intensity markedly drops when the laser power is higher than $\sim 67.5 \mathrm{~mW}(850 \mathrm{~nm})$. We identify this power as the laser damage threshold of (R-MPEA) $)_{1.5} \mathrm{PbBr}_{3.5}(\mathrm{DMSO})_{0.5}$ nano- wires, which is corresponding to a pump fluence of $\sim 0.52 \mathrm{~mJ}$ $\mathrm{cm}^{-2}\left(8.6 \times 10^{4} \mathrm{~W} \mathrm{~cm}{ }^{-2}\right)$, given the very small laser spot of $\sim 10 \mu \mathrm{m}$ in diameter.

To obtain more insight into the second-order NLO properties of these chiral perovskite materials, we carried out detailed polarization dependence measurements. Figure 4a summarizes the SHG intensity as function of the polarization angle of the linearly polarized pump at $850 \mathrm{~nm}$ from a horizontally oriented (R-MPEA) $)_{1.5} \mathrm{PbBr}_{3.5}(\mathrm{DMSO})_{0.5}$ single nanowire. The polarization plots follow a dipolar profile well fitted to a $\cos ^{4} \theta$ function ${ }^{52}$ with SHG maxima at polarization angles of about 65 and $245 \mathrm{deg}$, which should coincide with the dipolar axis of the ( $R$ - MPEA $)_{1.5} \mathrm{PbBr}_{3.5}(\mathrm{DMSO})_{0.5}$ nanowire. The dipolar axes of LHP materials have been suggested to be a result of the ionic displacement due to the distortion from ideal cubic perovskite $\mathrm{ABX}_{3}$ structure. ${ }^{30}$ In the case here, the displacement of $\mathrm{Br}$ with DMSO in the $\left[\mathrm{PbX}_{6}\right]$ octahedrons and the resulted structure distortion are the origins for the long-range polar nature of the chiral perovskite nanowires. The polarization ratio, defined as $\rho=\left(I_{\max }-I_{\min }\right) /$ $\left(I_{\max }+I_{\min }\right)$, was $(96.4 \pm 0.1) \%$ and $(72.4 \pm 0.1) \%$ for $p$ - and $s$-analyzers, respectively, which is considerably higher than that of the linear photoluminescence polarization of related LHP nanowires. ${ }^{54}$

We further replaced the $\lambda / 2$ plate with a $\lambda / 4$ plate (Figure 3a) to generate circularly polarized laser pump light to study the NLO chiroptical effects of these chiral perovskite nanowires. The $\lambda / 4$ plate was continuously rotated so that the fundamental pump was tuned from linear to left- and righthanded circular polarization, respectively. The results of the continuous polarization measurements, as shown in Figure $4 \mathrm{~b}$, reveal no mirror plane with respect to the $180^{\circ}$ rotation angle of the $\lambda / 4$ plate, which is a clear signature of chirality. ${ }^{55}$ The SHG circular dichroism (SHG-CD), defined as SHG-CD = 

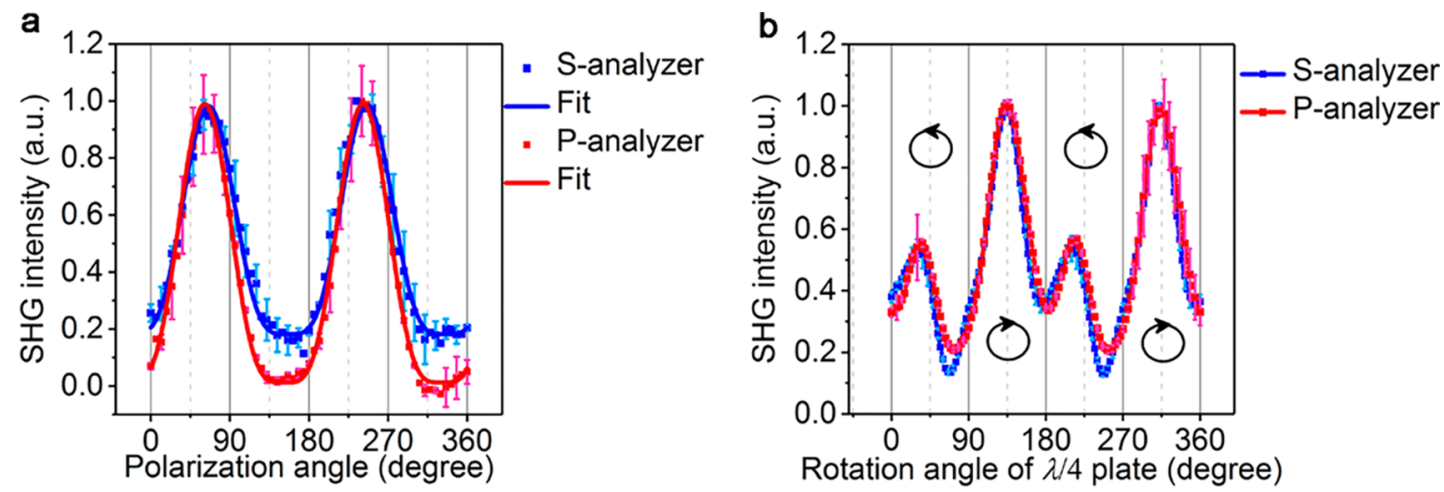

Figure 4. Polarization dependence of the SHG intensity from a single chiral perovskite nanowire. (a) SHG intensity from a horizontally oriented $(R \text {-MPEA })_{1.5} \mathrm{PbBr}_{3.5}(\mathrm{DMSO})_{0.5}$ nanowire as function of the linear polarization angle as tuned by the $\lambda / 2$ plate. The dots are the experimental data, while the solid lines are the $\cos ^{4} \theta$ fits. (b) SHG intensity from the nanowire as function of the rotation angle of the $\lambda / 4$ plate. The pump was lefthanded circularly polarized when the rotation angle was $45^{\circ}$ and $225^{\circ}$, and was right-handed circularly polarized when the rotation angle was $135^{\circ}$ and $315^{\circ}$, as indicated by the arrows. Excitation and detection wavelengths are 850 and $425 \mathrm{~nm}$, respectively. The error bars represent the variations between the measurements on the different samples, or the different sites on the same sample.

$\left(I_{\mathrm{RCP}}-I_{\mathrm{LCP}}\right) /\left(I_{\mathrm{RCP}}+I_{\mathrm{LCP}}\right)$, where $I_{\mathrm{RCP}}$ and $I_{\mathrm{LCP}}$ refer to the SHG intensity for the right- and left-circularly polarized pump, respectively, is a measure of the NLO chiroptical effect. ${ }^{55}$ The calculated SHG-CD for the (R- MPEA $)_{1.5} \mathrm{PbBr}_{3.5}(\mathrm{DMSO})_{0.5}$ nanowire was $(61.9 \pm 0.1) \%$ and $(74.0 \pm 0.1) \%$ for the $p$ - and $s$-out polarization, respectively.

Conclusions. In summary, we have reported a new type of 2D organic/inorganic hybrid lead halide perovskite materials with a chemical structure of (MPEA) $)_{1.5} \mathrm{PbBr}_{3.5}(\mathrm{DMSO})_{0.5}$ and an intrinsic noncentrosymmetric chiral crystal structure which exhibits strong second-order nonlinear optical responses. The introduction of the chiral $\beta$-methylphenethylammonium (MPEA) salt as the organic component induces the noncentrosymmetric assembly of the inorganic $2 \mathrm{D}$ perovskite layers. We fabricated nanowires from these new $2 \mathrm{D}$ chiral perovskite materials by the antisolvent assisted crystallization in a ternary solvent system, and identified the structure of this DMSO solvent intercalated perovskite phase. We observed the axial coordination of DMSO molecules with $\mathrm{Pb}^{2+}$ in the octahedron, which results in a distorted and partially edgeshared layered structure. The well-defined nanowires based on these new perovskite materials exhibited not only brilliant linear (broad band yellowish white light emission) but more importantly second-order nonlinear (SHG with high polarization ratios and chiroptical NLO effects) optical properties. Although the second-order nonlinear optical susceptibility of these chiral perovskites is not exceptionally high, our result is a proof-of-concept demonstration of the feasibility of realizing a second-order optical nonlinearity in hybrid organic/inorganic lead halide perovskite materials that might provide diverse opportunities for further rationally engineering the optoelectronic properties of LHP materials for technologically very important effects, such as ferroelectricity, pyroelectricity, piezoelectricity, and terahertz $(\mathrm{THz})$ generation.

\section{ASSOCIATED CONTENT}

\section{(S) Supporting Information}

The Supporting Information is available free of charge on the ACS Publications website at DOI: 10.1021/acs.nanolett.8b01616.

Experimental details, supporting figures and tables (PDF)

Crystal data for (R-MPEA $)_{1.5} \mathrm{PbBr}_{3.5}(\mathrm{DMSO})_{0.5}(\mathrm{CIF})$
Crystal data for (S-MPEA $)_{1.5} \mathrm{PbBr}_{3.5}(\mathrm{DMSO})_{0.5}$ (CIF)

\section{AUTHOR INFORMATION}

\section{Corresponding Author}

*E-mail: jialiang.xu@tju.edu.cn; jialiang.xu@nankai.edu.cn. ORCID

Jialiang Xu: 0000-0003-2441-4809

\section{Author Contributions}

C.Y. and X.L. contributed equally. C.Y. implemented the syntheses and characterizations of the chiral LHP materials. X.L. and S.S. carried out the NLO studies. J.L.X. conceived the idea, initiated, and supervised the project. Th.R., Y.Q.F., and S.S. cosupervised the project. All the authors contributed to analyzing the data and discussing on the results. The manuscript was written through contributions of all authors. All authors have given approval to the final version of the manuscript.

\section{Notes}

The authors declare no competing financial interest.

\section{ACKNOWLEDGMENTS}

This research was supported by the National Natural Science Foundation of China (21773168, 51503143, and 21761132007), Tianjin Natural Science Foundation (16JCQNJC05000), National Key R\&D Program of China (2016YFE0114900), "111 Project" of China (B18030), Innovation Foundation of Tianjin University (2016XRX0017), Tianjin 1000 Youth Talents Plan, Netherlands Organization for Scientific Research (NWO), including the Veni Grant (680-47-437), and Royal Netherlands Academy of Arts and Sciences (KNAW) with the China-Exchange Program (530-4CDP02). Jianbo Xiong and Dr. Paul Tinnemans are acknowledged for acquiring the SCXRD data. Chuangtang Wang, Dr. Huirong Su, and Prof. Lei Bi are acknowledged for determining the refractive indices of the crystals. A. Toonen is acknowledged for technical assistance. Prof. Shengxiong Xiao is acknowledged for the fruitful discussions.

\section{REFERENCES}

(1) Li, W.; Wang, Z.; Deschler, F.; Gao, S.; Friend, R. H.; Cheetham, A. K. Nat. Rev. Mater. 2017, 2, 16099. 
(2) Sutherland, B. R.; Sargent, E. H. Nat. Photonics 2016, 10, 295302.

(3) Manser, J. S.; Christians, J. A.; Kamat, P. V. Chem. Rev. 2016, 116, 12956-13008.

(4) Kovalenko, M. V.; Protesescu, L.; Bodnarchuk, M. I. Science 2017, 358, 745-750.

(5) Kim, Y.-H.; Cho, H.; Lee, T.-W. P. Proc. Natl. Acad. Sci. U. S. A. 2016, 113, 11694-11702.

(6) Stranks, S. D.; Snaith, H. J. Nat. Nanotechnol. 2015, 10, 391402.

(7) Park, N.-G.; Grätzel, M.; Miyasaka, T.; Zhu, K.; Emery, K. Nat. Energy 2016, 1, 16152.

(8) Sun, Z.; Liu, X.; Khan, T.; Ji, C.; Asghar, M. A.; Zhao, S.; Li, L.; Hong, M.; Luo, J. Angew. Chem., Int. Ed. 2016, 55, 6545-6550.

(9) Hwang, J.; Rao, R. R.; Giordano, L.; Katayama, Y.; Yu, Y.; ShaoHorn, Y. Science 2017, 358, 751-756.

(10) Ahmadi, M.; Wu, T.; Hu, B. Adv. Mater. 2017, 29, 1605242.

(11) Wang, H.; Kim, D. H. Chem. Soc. Rev. 2017, 46, 5204-5236.

(12) Veldhuis, S. A.; Boix, P. P.; Yantara, N.; Li, M.; Sum, T. C.; Mathews, N.; Mhaisalkar, S. G. Adv. Mater. 2016, 28, 6804-6834.

(13) Pawlicki, M.; Collins, H. A.; Denning, R. G.; Anderson, H. L. Angew. Chem., Int. Ed. 2009, 48, 3244-3266.

(14) Clark, J.; Lanzani, G. Nat. Photonics 2010, 4, 438-446.

(15) Gieseking, R. L.; Mukhopadhyay, S.; Risko, C.; Marder, S. R.; Brédas, J.-L. Adv. Mater. 2014, 26, 68-84.

(16) Tokel, O.; Turnalı, A.; Makey, G.; Elahi, P.; Çolakoğlu, T.; Ergeçen, E.; Yavuz, Ö.; Hübner, R.; Zolfaghari Borra, M.; Pavlov, I.; Bek, A.; Turan, R.; Kesim, D. K.; Tozburun, S.; Ilday, S.; Ilday, F. Ö. Nat. Photonics 2017, 11, 639-645.

(17) Yue, S.; Slipchenko, M.; Cheng, J. Laser \& Photon. Rev. 2011, 5, 496-512.

(18) Dutto, D.; Raillon, K.; Schenk, K.; Radenovic, A. Nano Lett. 2011, 11, 2517-2521.

(19) Cai, F.; Yu, J.; Qian, J.; Wang, Y.; Chen, Z.; Huang, J.; Ye, Z.; He, S. Laser Photonics Rev. 2014, 8, 865-874.

(20) Yu, H.; Wu, H.; Pan, S.; Yang, Z.; Hou, X.; Su, X.; Jing, Q.; Poeppelmeier, K. R.; Rondinelli, J. M. J. Am. Chem. Soc. 2014, 136, 1264-1267.

(21) Kim, E.; Steinbrück, A.; Buscaglia, M. T.; Buscaglia, V.; Pertsch, T.; Grange, R. ACS Nano 2013, 7, 5343-5349.

(22) Timpu, F.; Hendricks, N. R.; Petrov, M.; Ni, S.; Renaut, C.; Wolf, H.; Isa, L.; Kivshar, Y.; Grange, R. Nano Lett. 2017, 17, 53815388.

(23) Stoumpos, C. C.; Frazer, L.; Clark, D. J.; Kim, Y. S.; Rhim, S. H.; Freeman, A. J.; Ketterson, J. B.; Jang, J. I.; Kanatzidis, M. G. J. Am. Chem. Soc. 2015, 137, 6804-6819.

(24) Walters, G.; Sutherland, B. R.; Hoogland, S.; Shi, D.; Comin, R.; Sellan, D. P.; Bakr, O. M.; Sargent, E. H. ACS Nano 2015, 9, 9340-9346.

(25) Zhang, R.; Fan, J.; Zhang, X.; Yu, H.; Zhang, H.; Mai, Y.; Xu, T.; Wang, J.; Snaith, H. J. ACS Photonics 2016, 3, 371-377.

(26) Kalanoor, B. S.; Gouda, L.; Gottesman, R.; Tirosh, S.; Haltzi, E.; Zaban, A.; Tischler, Y. R. ACS Photonics 2016, 3, 361-370.

(27) Saouma, F. O.; Stoumpos, C. C.; Wong, J.; Kanatzidis, M. G.; Jang, J. I. Nat. Commun. 2017, 8, 742.

(28) Wang, C.; Zhang, T.; Lin, W. Chem. Rev. 2012, 112, 10841104.

(29) Marder, S. R.; Sohn, J. E.; Stucky, G. D. Materials for Nonlinear Optics: Chemical Perspectives; American Chemical Society: Washington, DC, 1991; Vol.455.

(30) Rakita, Y.; Bar-Elli, O.; Meirzadeh, E.; Kaslasi, H.; Peleg, Y.; Hodes, G.; Lubomirsky, I.; Oron, D.; Ehre, D.; Cahen, D. P. Proc. Natl. Acad. Sci. U. S. A. 2017, 114, E5504-E5512.

(31) G, S.; Mahale, P.; Kore, B. P.; Mukherjee, S.; Pavan, M. S.; De, C.; Ghara, S.; Sundaresan, A.; Pandey, A.; Guru Row, T. N.; Sarma, D. D. J. Phys. Chem. Lett. 2016, 7, 2412-2419.

(32) Liao, W.-Q.; Zhang, Y.; Hu, C.-L.; Mao, J.-G.; Ye, H.-Y.; Li, P.F.; Huang, S. D.; Xiong, R.-G. Nat. Commun. 2015, 6, 7338.
(33) You, Y.-M.; Liao, W.-Q.; Zhao, D.; Ye, H.-Y.; Zhang, Y.; Zhou, Q.; Niu, X.; Wang, J.; Li, P.-F.; Fu, D.-W.; Wang, Z.; Gao, S.; Yang, K.; Liu, J.-M.; Li, J.; Yan, Y.; Xiong, R.-G. Science 2017, 357, 306-309.

(34) Yan, R.; Gargas, D.; Yang, P. Nat. Photonics 2009, 3, 569-576.

(35) Dasgupta, N. P.; Sun, J.; Liu, C.; Brittman, S.; Andrews, S. C.; Lim, J.; Gao, H.; Yan, R.; Yang, P. Adv. Mater. 2014, 26, 2137-2184.

(36) Dou, L.; Wong, A. B.; Yu, Y.; Lai, M.; Kornienko, N.; Eaton, S. W.; Fu, A.; Bischak, C. G.; Ma, J.; Ding, T.; Ginsberg, N. S.; Wang, L.W.; Alivisatos, A. P.; Yang, P. Science 2015, 349, 1518-1521.

(37) Yuan, M.; Quan, L. N.; Comin, R.; Walters, G.; Sabatini, R.; Voznyy, O.; Hoogland, S.; Zhao, Y.; Beauregard, E. M.; Kanjanaboos, P.; Lu, Z.; Kim, D. H.; Sargent, E. H. Nat. Nanotechnol. 2016, 11, 872-877.

(38) Liu, W.; Xing, J.; Zhao, J.; Wen, X.; Wang, K.; Lu, P.; Xiong, Q. Adv. Opt. Mater. 2017, 5, 1601045.

(39) Chen, S.; Shi, G. Adv. Mater. 2017, 29, 1605448.

(40) Billing, D. G.; Lemmerer, A. CrystEngComm 2006, 8, 686-695.

(41) Ahn, J.; Lee, E.; Tan, J.; Yang, W.; Kim, B.; Moon, J. Mater. Horiz. 2017, 4, 851-856.

(42) Shi, D.; Adinolfi, V.; Comin, R.; Yuan, M.; Alarousu, E.; Buin, A.; Chen, Y.; Hoogland, S.; Rothenberger, A.; Katsiev, K.; Losovyj, Y.; Zhang, X.; Dowben, P. A.; Mohammed, O. F.; Sargent, E. H.; Bakr, O. M. Science 2015, 347, 519-522.

(43) Jeon, N. J.; Noh, J. H.; Kim, Y. C.; Yang, W. S.; Ryu, S.; Seok, S. I. Nat. Mater. 2014, 13, 897.

(44) Dohner, E. R.; Jaffe, A.; Bradshaw, L. R.; Karunadasa, H. I. J. Am. Chem. Soc. 2014, 136, 13154-13157.

(45) Yuan, Z.; Zhou, C.; Tian, Y.; Shu, Y.; Messier, J.; Wang, J. C.; van de Burgt, L. J.; Kountouriotis, K.; Xin, Y.; Holt, E.; Schanze, K.; Clark, R.; Siegrist, T.; Ma, B. Nat. Commun. 2017, 8, 14051.

(46) Schulz, M.; Zablocki, J.; Abdullaeva, O. S.; Brück, S.; Balzer, F.; Lützen, A.; Arteaga, O.; Schiek, M. Nat. Commun. 2018, 9, 2413.

(47) Miles, A. J.; Wallace, B. A. Chem. Soc. Rev. 2016, 45, 48594872.

(48) Hu, T.; Smith, M. D.; Dohner, E. R.; Sher, M.-J.; Wu, X.; Trinh, M. T.; Fisher, A.; Corbett, J.; Zhu, X. Y.; Karunadasa, H. I.; Lindenberg, A. M. J. Phys. Chem. Lett. 2016, 7, 2258-2263.

(49) Cortecchia, D.; Neutzner, S.; Srimath Kandada, A. R.; Mosconi, E.; Meggiolaro, D.; De Angelis, F.; Soci, C.; Petrozza, A. J. Am. Chem. Soc. 2017, 139, 39-42.

(50) Smith, M. D.; Karunadasa, H. I. Acc. Chem. Res. 2018, 51, 619627.

(51) He, J.; Zeller, M.; Hunter, A. D.; Xu, Z. J. Am. Chem. Soc. 2012, $134,1553-1559$

(52) Duan, Y.; Ju, C.; Yang, G.; Fron, E.; Coutino-Gonzalez, E.; Semin, S.; Fan, C.; Balok, R. S.; Cremers, J.; Tinnemans, P.; Feng, Y.; Li, Y.; Hofkens, J.; Rowan, A. E.; Rasing, T.; Xu, J. Adv. Funct. Mater. 2016, 26, 8968-8977.

(53) Sutherland, R. L. In Handbook of Nonlinear Optics; Thompson, B. J., Ed.; Marcel Dekker, Inc.: New York, 1996.

(54) Täuber, D.; Dobrovolsky, A.; Camacho, R.; Scheblykin, I. G. Nano Lett. 2016, 16, 5087-5094.

(55) Sioncke, S.; Verbiest, T.; Persoons, A. Mater. Sci. Eng., R 2003, $42,115-155$. 